Schilddrüse 1997 



\section{Schilddrüse 1997}

Henning-Symposium

\section{Iod und Schilddrüse}

13. Konferenz über die menschliche Schilddrüse Heidelberg

Wissenschaftliche Fortbildungsveranstaltung der Sektion Schilddrüse der Deutschen Gesellschaft für Endokrinologie unter Beteiligung der

Chirurgischen Arbeitsgemeinschaft Endokrinologie der Deutschen Gesellschaft für Chirurgie - CAEK der

Arbeitsgemeinschaft Schilddrüse der Deutschen Gesellschaft für Nuklearmedizin und der

Sektion Angewandte Endokrinologie der Deutschen Gesellschaft für Endokrinologie

Herausgegeben von

Chr. Reiners · B. Weinheimer

\footnotetext{
$W$
$\mathrm{DE}$
$\mathrm{G}$

Walter de Gruyter Berlin - New York 1998
} 


\section{Herausgeber}

Prof. Dr. med. Chr. Reiners

Direktor der Abteilung für Nuklearmedizin

Klinikum der Universität Würzburg

Josef-Schneider-Straße 2

97080 Würzburg

Dr. B. Weinheimer

Karlsbergstraße 2

66424 Homburg/Saar

Die Deutsche Bibliothek - CIP Einheitsaufnahme

Schilddrüse 1997 : Iod und Schilddrüse ; wissenschaftliche Fortbildungsveranstaltung der Sektion Schilddrüse der Deutschen Gesellschaft für Endokrinologie unter Beteiligung der Chirurgischen Arbeitsgemeinschaft Endokrinologie der Deutschen Gesellschaft für Chirurgie, CAEK, der Arbeitsgemeinschaft Schilddrüse der Deutschen Gesellschaft für Nuklearmedizin und der Sektion Angewandte Endokrinologie der Deutschen Gesellschaft für Endokrinologie / 13. Konferenz über die Menschliche Schilddrüse, Heidelberg, Henning-Symposium. Hrsg. von Chr. Reiners ; B. Weinheimer. - Berlin ; New York : de Gruyter, 1998 ISBN 3-11-016327-6

Copyright 1998 by Walter de Gruyter GmbH \& Co., D-10785 Berlin

Dieses Werk einschließlich aller seiner Teile ist urheberrechtlich geschützt. Jede Verwertung außerhalb der engen Grenzen des Urheberrechtsgesetzes ist ohne Zustimmung des Verlages unzulässig und strafbar. Das gilt insbesondere für Vervielfältigungen, Übersetzungen, Mikroverfilmungen und die Einspeicherung und Verarbeitung in elektronischen Systemen.

Der Verlag hat für die Wiedergabe aller in diesem Buch enthaltenen Informationen (Programme, Verfahren, Mengen, Dosierungen, Applikationen etc.) mit Autoren bzw. Herausgebern große Mühe darauf verwandt, diese Angaben genau entsprechend dem Wissensstand bei Fertigstellung des Werkes abzudrucken. Trotz sorgfältiger Manuskripterstellung und Korrektur des Satzes können Fehler nicht ganz ausgeschlossen werden. Autoren bzw. Herausgeber und Verlag übernehmen infolgedessen keine Verantwortung und keine daraus folgende oder sonstige Haftung, die auf irgendeine Art aus der Benutzung der in dem Werk enthaltenen Informationen oder Teilen davon entsteht.

Die Wiedergabe von Gebrauchsnamen, Handelsnamen, Warenbezeichnungen und dergleichen in diesem Buch berechtigt nicht zu der Annahme, daß solche Namen ohne weiteres von jedermann benutzt werden dürfen. Vielmehr handelt es sich häufig um gesetzlich geschützte, eingetragene Warenzeichen, auch wenn sie nicht eigens als solche gekennzeichnet sind.

Satz: Ditta Ahmadi, Berlin.

Druck: Gericke GmbH, Berlin.

Buchbinderische Verarbeitung: Lüderitz \& Bauer, Berlin.

Umschlagentwurf: Rudolf Hübler, Berlin.

Printed in Germany. 